\title{
Pediatric Orthopaedics in Precarious Environment Possibilities and Limits of Humanitarian Surgery
}

\author{
Michel Onimus \\ Faculty of Medicine, Franch County University, Besançon, France \\ Email address: \\ monimus@wanadoo.fr \\ To cite this article: \\ Michel Onimus. Pediatric Orthopaedics in Precarious Environment Possibilities and Limits of Humanitarian Surgery. Journal of Surgery. \\ Vol. 8, No. 3, 2020, pp. 97-103. doi: 10.11648/j.js.20200803.14
}

Received: May 8, 2020; Accepted: May 25, 2020; Published: June 4, 2020

\begin{abstract}
This is a retrospective study concerning a 35 years experience of pediatric orthopedic humanitarian surgery performed in precarious situation. This account may be useful to share with younger surgeons considering initiating such a surgery in developing countries. The data of 80 surgical missions held between 1983 and 2018 in Central African Republic have been collected and analyzed. The missions were planned with authorization of the local ministry of health. Their duration was usually two weeks, one week in the capital and one week in about fifteen villages in the country having small rehabilitation centers created when poliomyelitis was devastating. Surgery was performed in the local health center or hospital, with cooperation of the local medical team, sometimes without electricity, usually without radiograph, allowing only clinical diagnosis and not aggressive surgery. Postoperative care was given in the rehabilitation center. All in all 7500 children were seen in out-patients clinics and 2200 underwent surgery. Sequelae of poliomyelitis which included $66 \%$ of out-patients between 1983 and 2000 turned down and became very rare, replaced by a more varied pathology with predominantly congenital malformations and sequelae of intramuscular injections, as well as sequelae of burns and sequelae of neurologic suffering (cerebral palsy, neuromalaria, sequelae of poisoning by toxic cassava). These pathologies are analyzed and the therapeutic options are discussed considering the local facilities. The families often neglected the given appointments, and due to this poor cooperation $25 \%$ only out of the operated patients were reviewed, making ineffective any statistical analysis. Nevertheless numerous pieces of information concerning the postoperative result and the late follow-up could be obtained from the local teams, allowing a global evaluation of the validity of this kind of humanitarian surgery.
\end{abstract}

Keywords: Poliomyelitis, Humanitarian Surgery, Sequelae of Intramuscular Injections, Orthopedic Surgery in Precarious Environment

\section{Introduction}

The humanitarian surgery can be performed in various conditions, according to the human and material context in which it is carried out. It may be divided in emergency surgery with specific needs and in so-called surgery of assistance to development; pediatric orthopedics fall within the context of the latter. It takes different aspects according to the place where it is performed, according to the local equipments and according to the financial investment considered. On the basis of an experience of 35 years of pediatric orthopedic surgery performed in precarious environment, the author analyzes the pathology observed and the changes which occurred with time, as well as the surgical possibilities and the results. Lastly therapeutic options are discussed according to the local context.

\section{Material and Methods}

This is a retrospective study of 80 pediatric orthopedic surgical missions held in Central African Republic between 1983 and 2018, planned with agreement of the local Ministry of health. The initial purpose was to take in care the children presenting with sequelae of poliomyelitis. The duration of the missions was usually 2 weeks, one week in the capital with acceptable facilities and one week in the countryside, in about fifteen small cities or villages equipped with small rehabilitation centers created during the 1970-1980 years, when poliomyelitis was devastating. The staffs in these centers (missionary nuns, physiotherapists.) were our 
essential local partners. Surgery was performed in the local health center or hospital in cooperation with the local medical team for whom a significant training program was set on. The basic principle was to do surgery with the means at disposal of the local surgeons, at low cost, using simple and easily reproducible protocols, without bringing sophisticated techniques, and planning basic postoperative care, as much as possible restricted to the cast removal, the dressing change and the rehabilitation. Patients assessment was only clinical; the only possible surgery had to be non aggressive and restricted to the limbs. No osteosynthesis was available, forbidding any bone surgery on the hip. Maintaining the operative correction was entrusted to the postoperative casts, whose making was an important step of the procedure. Whenever possible, surgery was performed under haemostasis with Esmarch tourniquet. Anaesthesias were conducted following local protocols, using Diazepam, Ketamin, Atropin, without intubation (except for cleft lip closure). Caudal anaesthesia was used until 6 or 7 years of age and spinal anaesthesia was performed from 12 or 13 years of age. Initially the main pathology was sequelae of poliomyelitis which disappeared more or less completely with vaccination campaigns and allowed emergence of a more diversified pathology, including congenital malformations (mainly club foot), sequelae of trauma, sequelae of bone or joint infections, axial deviations of the lower limbs, sequelae of burns, sequelae of intramuscular injections.

Table 1. Pathologies observed between 2010 et 2017 (total out-patients: 2800).

\begin{tabular}{ll}
\hline Congenital malformations & $22 \%$ \\
\hline Club foot & $10 \%$ \\
Cleft lips: & $5 \%$ \\
Aplasias: & $1 \%$ \\
Varias: & $6 \%$ \\
Sequalae of injections & $16 \%$ \\
Sciatic Paralysis: & $7 \%$ \\
Nécrosis of femoral head: & $1 \%$ \\
Knee stiffness: & $8 \%$ \\
Neurologic sequelae & $15 \%$ \\
Sequelae of trauma & $14 \%$ \\
Sequelae of infection of bone or joint & $7 \%$ \\
Axial deviations & $6 \%$ \\
Sequelae of burns & $5 \%$ \\
Sequelae of poliomyelitis & $3 \%$ \\
Erb Duchenne paralysis & $2 \%$ \\
Pott's kyphosis & $1 \%$ \\
Varia & $9 \%$ \\
\hline
\end{tabular}

Congenital malformations varias: syndactylism, congenital hypertrophies, myelomeningocele, radial club hand.

\section{Results}

\subsection{Out-Patients Clinics}

7500 patients were examined during the missions, and 2 200 were operated on. Between the years 1983 and 2000 sequelae of poliomyelitis were predominant in the clinics (66\% of the patients); afterwards the number dropped and became very infrequent (7\% between 2000 and 2010 and $2 \%$ between 2010 and 2018) and concerned mainly formerly affected patients. Since 2010 the most frequently observed pathologies were congenital malformations (22\%), sequelae of intramuscular injections (16\%), sequelae of neurologic attack (15\%), sequelae of trauma (14\%) (Table 1).

\subsection{Surgery}

Until the year 2000 the most frequently performed surgeries concerned sequelae of poliomyelitis (70\%); since the year 2000 the figure is $1 \%$ and the most frequent surgeries concern congenital malformations (38\%) and sequelae of intramuscular injections (15\%) (Table 2).

Table 2. Operations performed since 2011 (Total: 850 operations).

\begin{tabular}{ll}
\hline Club foot: & $25 \%$ \\
Postero-medial release: & $12 \%$ \\
Protocole of Ponseti: & $5 \%$ \\
Triple arthrodesis: & $7 \%$ \\
Talectomy: & $1 \%$ \\
Cleft lips & $8 \%$ \\
Other congenital malformations & $5 \%$ \\
Sequalae of injection & $15 \%$ \\
Tibial posterior transfert & $6 \%$ \\
Quadriceps release & $9 \%$ \\
Osteotomies for axial deviations & $11 \%$ \\
Tenotomies for spasticity & $12 \%$ \\
Sequelae of burns & $10 \%$ \\
Chronic osteitis & $4 \%$ \\
Sequelae of trauma & $1 \%$ \\
Sequelae of poliomyelitis & $1 \%$ \\
Varia & $8 \%$ \\
\hline
\end{tabular}

Complications: 4 deaths occurred, all of them during too «aggressive» anaesthesias performed by local incompetent anesthesiologists. Septic complications were observed mainly in two situations: after subtalar and mediotarsal triple arthrodesis (10 cases out of 160 , i.e. $6 \%$ ) and complicating hematomas after wide quadriceps release (23 cases out of 150 , i.e. $15 \%)$.

Because of the nature of the missions and because of the lack of cooperation from the families only $25 \%$ of the operated patients were reviewed, preventing any statistical analysis; however information concerning their postoperative condition was obtained in numerous cases, giving possibility of an overall evaluation of this activity.

\section{Discussion}

\subsection{The Distinctive Features of Pediatric Orthopaedic Surgery}

The advantage of this surgery is that, at least for a large part of it, it can be performed in a basic or simple way, without complicated or sophisticated means and equipments, allowing to be considered in precarious conditions and performed at low cost. Another advantage of the pediatric orthopaedic surgery is that it is an elective surgery. It may be scheduled beforehand with local partners who plan the missions, inform the population, screen and list the crippled 
children and gather them for the mission. These local partners are essential as well for ensuring the postoperative care, performing postoperative physiotherapy, removing the casts, making the orthotics when necessary, and reviewing and evaluating the patients.

There is no public screening and the lesions are commonly seen at a very advanced stage, with accentuated and poorly reducible deformities, making the surgery more difficult. Otherwise the families are poorly compliant and scarcely respect the prescriptions; follow-up appointments are frequently forgotten; the children are not very carefully watched and the postoperative casts are rapidly damaged, especially during the wet season.

A decentralized surgery has the advantage to make the access to medical care easier for the families, avoiding complicated trips (in Africa the mother stays besides her child during all the hospitalization time, and she has to leave the family maintenance and the field cultivation for sometimes several weeks). Working in the local health center has the advantage to implicate local doctors and nursing staff in the postoperative care. An important attempt at teaching and training was done with the staff during the clinics and the surgical sessions, but with time an almost total failure must be admitted, maybe due to weak motivations from the health professionals.

The disadvantages of this decentralized surgery, performed in precarious conditions, include septic risk, precluding any osteosynthesis, and the impossibility to start long protocols such as the limbs equalization. Another disadvantage is to have to adapt oneself in each new situation. Despite the basic operative rooms fitting and despite the frequent poor quality of the asepsis, septic complications were infrequent; this may be due to short procedures and to a strictly performed « no touch technique ».

\subsection{The Sequelae of Poliomyelitis}

They made up the main part of the surgery before the year 2000. Because of the above-mentioned reasons, surgery of limbs inequality was excluded as well as surgery of the dislocated or unstable hip. Limbs inequalities are mainly observed in unilateral paralysis and in these situations standing and walking are possible with a stick or a crutch (figure 1). Moreover the affected limb is usually completely paralyzed and hip instability may be associated; any attempt to put weight on it is illusive, because the child is already walking and will not accept the constraint of an orthosis. So indications for surgery in monoplegia are not frequent and may be considered more for cosmetic than functional purpose, in order to correct unattractive hip or knee flexions contractures. Therefore surgery is advised mainly in bilateral sequelae, with no or mild inequality, the child commonly moving by crawling or in squatting position (figure 2) as described by Cross [1]. The aim is to give the child a standing and walking ability with or without caliper or crutches. Prognosis for ability of walking should be established during the preoperative evaluation according to the muscle assessment.

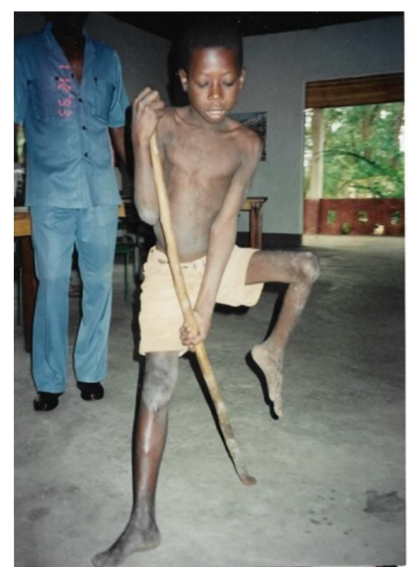

Figure 1. Monoplegia post-poliomyelitis. What should be done? The left lower limb is completely paralyzed with a $90^{\circ}$ hip flexion contracture and considerable shortness. The child is already able to walk with a stick. There is no possibility to load the limb. The indication for surgery is more to improve the cosmetic aspect than to improve walking. A crutch was advised (but will be more costly than a stick when renewal, and maybe will be given up.).

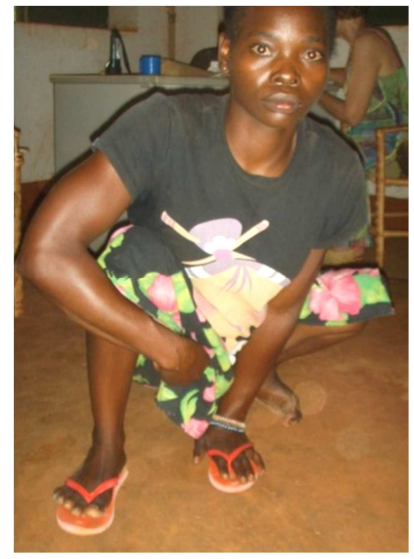

Figure 2. Post polio paraplegia. There are severe contractures of both hips and knees. The patient is moving in squatting position, wearing flips-flops on the right foot and the left hand. Surgical correction of the contractures may be considered, but the treatment will be long-lasting because of the age of the patient and because of the severity of deformations.

Only few and simple surgical protocols were used: on the hip, correction of the flexion contracture by hip flexors tenotomy (sartorius, rectus femoris, tensor of the fascia lata extended laterally according to the degree of abductum of the hip); on the knee correction of the flexion contracture by hamstring muscles and fascia lata tenotomy); on the foot correction of equinismus deformity by Achilleus tendon lengthening, correction of equinovarus deformity by triple arthrodesis. Postoperative rehabilitation follows a rigorous protocol: the correction of the hip contracture is rapidly obtained in a few days by immediate postoperative posture in prone position, then by daily postures and mobilizations. The correction of the knee contracture is obtained more progressively, using hand-crafted articulated splints included in the cast, allowing daily mobilization of the knee and progressive painless and harmless correction of the contracture (figure 3). Using this protocol a complete correction can be obtained in a few days or a few weeks, 
avoiding any risk of neurovascular complications and keeping full range of motion. Additional surgeries as capsulotomy or skin release were never necessary. In a previous study [2] 70\% satisfying results were observed, assessed on the ability for the child to go to school. Occasionally when the quadriceps muscle is paralyzed, a mild knee flexion contracture may require an orthosis for stabilization of the knee during walking, whereas gluteus magnus and triceps muscles are powerful and could be sufficient for knee stabilization. In such a situation the correction of the contracture by supracondylar osteotomy, giving a mild overcorrection in extension, makes the contraction of the gluteus magnus and triceps muscles sufficient to stabilize the knee, making the orthosis useless. One should remember that children will accept orthosis only if it improves function and is felt as beneficial.

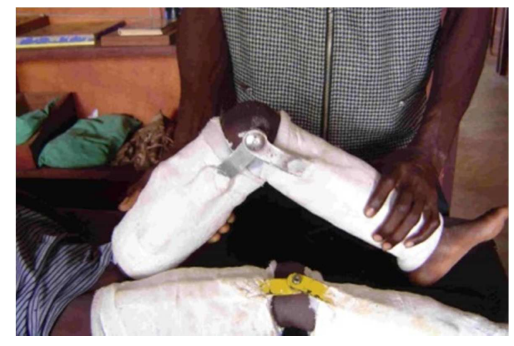

Figure 3. Apparatus for progressive correction of the knee flexion contracture. The metallic splints are included in the plaster; they allow a mobilization of the knee and daily correction of a few degrees of the flexion contracture. The correction is very progressive and harmless.

\subsection{Congenital Malformations}

Congenital malformations are frequent, especially equinovarus club foot (25\% of surgery since 2000 (Table 2). Postero-medial release is difficult because of the stiffness and severity of the deformity and the correction may be incomplete in spite of a wide release including a complete capsulotomy of the medial aspect of the subtalar joint. About 20 years ago the Ponseti conservative protocol [3] was started, but the results of the treatment according to Ponseti, i.e. started as soon as possible after birth, were slightly disappointing, mainly because of the lack of cooperation from the families, reticent to pay the orthosis cost and hardly accepting a long-term contention. Non compliance with bracing has already been recognized as a major factor of recurrence [4]. In our experience it is more profitable to start later the treatment by successive casts, namely in the few months preceding walking, in order to have the treatment completed when the baby begins standing and walking, ensuring a better stability to the correction and making the orthosis less necessary. Then, when started lately the results after correction by casts may be imperfect and a posteromedial release may be necessary, but surgery is always significantly facilitated by the relaxation given by the casts. A talectomy was performed in 7 cases with very severe and stiff deformities in children between 5 and 9 years of age. Five of them were reviewed: the clinical aspect was correct, with a plantigrade position of the foot, but walking was without a normal progress of the step.

Treatment of tibial or fibular aplasia is challenging. Because of the usual inequality we did not consider any surgery in unilateral cases, except by suggesting amputation which is rarely accepted. In two cases of incomplete bilateral tibial aplasia, a tibialisation of the fibula was performed with a double stage surgery. The valgus deformity of fibular aplasia was corrected by tibial varus osteotomy and Achilleus tendo lengthlening, in order to facilitate prosthesis.

\subsection{Sequelae of Intramuscular Injection}

These sequelae are frequent and endemic, counting for up to $16 \%$ of out-patients; they are still a burden and should be prevented with policy changes and education regarding injection practices [5]. In Central Africa, the injection of quinine salts is still the most used treatment of malaria in children, because being the cheapest one and because available everywhere. But the injection is frequently wrongly performed:

\subsubsection{Too Medial Injection}

When too medially performed the injection can induce a partial paralysis of the sciatic nerve, usually compromising the fibers of the common fibular nerve resulting in an equinovarus deformity of the foot. The tibial posterior muscle is usually not affected and may be transferred on the dorsum of the foot, sutured to the peroneal anterior muscle if present or more frequently on the periosteum of the 4th metatarsal base [6]. The results of the tibial posterior transfer were evaluated on 30 reviewed cases: they were acceptable in $50 \%$ of cases with contraction giving a right angle position to the foot and unsatisfying in $50 \%$ with a contraction not or poorly effective. An irreducible equinovarus deformity requires a triple subtalar and midtarsal arthrodesis.

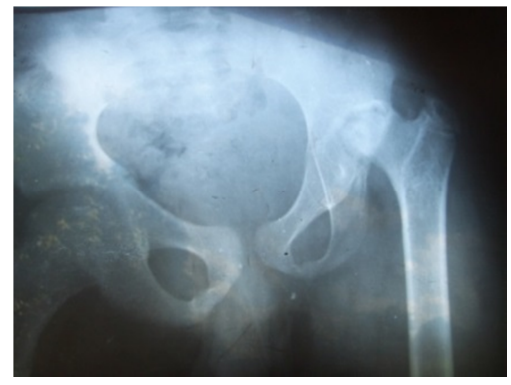

Figure 4. Necrosis of the femoral head in a 13 years old patient, reporting an history of intragluteal injection for malaria attack 4 years before. There was no other previous history. The diagnosis of post-injection necrosis was considered.

\subsubsection{Too Deep Injection}

When the injection is performed too deeply in the buttock it may be performed into the hip joint, resulting in a necrosis of the femoral head with limb shortening and a painful and stiff hip. Several cases of femoral head necrosis in children were observed, confirmed by X-Rays, without apparent etiology apart from a history of gluteal injection (figure 4), unfortunately without possible treatment. 


\subsubsection{Intraquadricipital Injection}

In babies and young children the injection is preferably made on the anterior aspect of the thigh in the quadriceps muscle and it can induce a fibrosis of the muscle, resulting in a complete stiffness of the knee in full extension, sometimes even in a knee recurvatum (figure 5). These sequelae are especially harmful when bilateral. Surgical treatment requires a complete release of the quadriceps muscle according to Judet [7], but eletrocautery is necessary and therefore this surgery is not feasible everywhere. A full range of flexion of the knee is easily obtained if the muscle retraction is limited to the vastus lateralis muscle, making the release easier, but more frequently the retraction includes the vastus medialis muscle and the release is more difficult and should be cautious because of the vicinity of the vessels. Immediate results are usually excellent with a full range of flexion, but the long-term result is closely dependant on the quality of the postoperative reeducation (preservation of the range of motion and quadriceps muscle reinforcement), and in more than $50 \%$ of cases we observed a significant secondary decrease of flexion with a residual range of flexion $0^{\circ}-45^{\circ}$, nevertheless allowing an acceptable walking. Despite a careful hemostasis, a postoperative septic hematoma occurred in $15 \%$ of cases, compromising the final result. Due to the lack of elasticity of the skin in front of the patella, skin necrosis may occur when a full range of motion is achieved and the reeducation should be cautious [8]. Proximal [9] or distal [10] quadricepsplasty were suggested instead of the muscle release; however this requires a few weeks immobilization in $50^{\circ}-60^{\circ}$ flexion, and lengthening of the distal quadriceps tendon may result in weakness of the muscle, although in Fiogbe series the results of muscle tests were at least $3 / 5$ in all cases [11]. Occasionally the rectus femoris muscle is the only muscle involved and the knee stiffness is apparent only with the hip fully extended. Discomfort is moderate and indication for surgery should be carefully discussed, either by proximal tendon release or preferently by middle shaft release of the muscle [12].

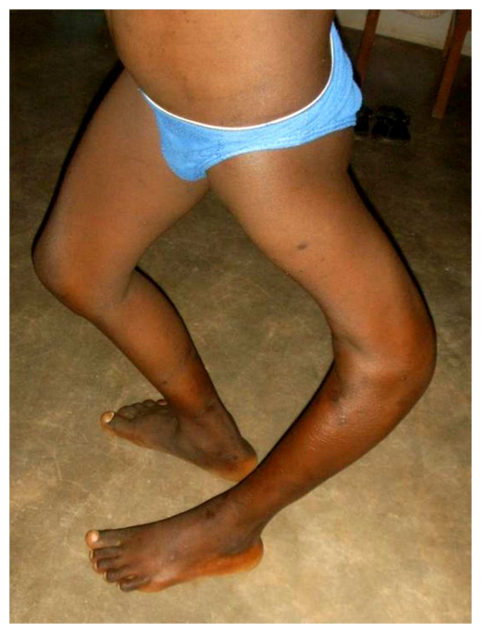

Figure 5. Considerable left genu recurvatum occurring after intraquadricipital injection of quinine salts. There is no paralysis. The recurvatum is due to the retractile fibrosis of the muscle caused by the toxicity of the quinine. Only a few degrees of mobility are possible.

\subsection{Cleft lips}

The closure of cleft lips was performed using the Delaire procedure [13], not dissimilar from that of Millard, with wide subperiosteal undermining over the maxilla, the normal reinsertion of the naso-labial muscles providing a nasal symmetry [14, 15]. Intubation is necessary, restricting this surgery to hospitals where oxygen was available. Postoperative course was uneventful despite a poor collaboration from the families (the children beginning sometimes to eat as soon as the day following the operation.). Cleft lips are relatively frequent malformations, but ignored, the children being hidden in the house and socially poorly integrated.

\subsection{Sequelae of Burns}

Burns are very frequent in children because the cooking fire is made on the ground and kids play and run all around and may fall in the fire, especially when epileptics. Sequelae are predominantly seen at the hand, with skin contractures compromising the joints motion [16] (figure 6). Correction is achieved by dividing the skin contracture and covering the skin gap with a full thickness skin graft taken on the abdomen [17]. This is easily performed with a knife and a forceps (according to the principle of using the local facilities.). The abdominal skin gap is closed, avoiding further numerous painful and costly dressings. In the absence of local abnormal signs the first dressing is made on the 21 th postoperative day and motion and rehabilitation is started.

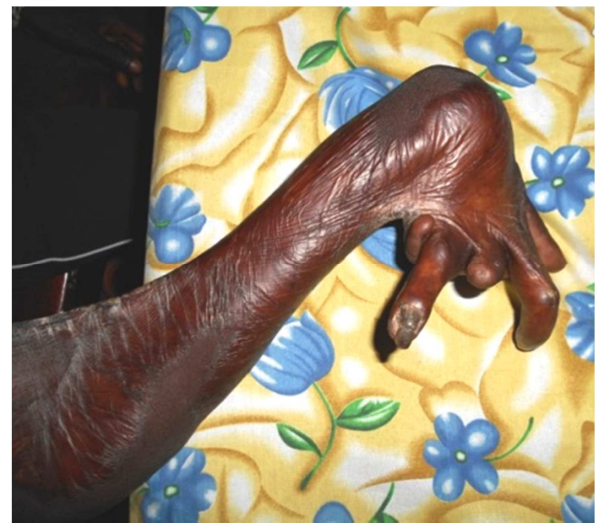

Figure 6. Severe sequelae of burns on the right hand, with considerable lateral tilting of the wrist on ulnar side and multiple fingers distortions.

\subsection{Axial Deviations of Lower Limbs}

Deviations in genu valgum or genu valgum are frequent; their etiology is imprecise, maybe due to unrecognized rickets, worsened by nutritional factors (in Central Africa food is essentially cassava, well-known to have no nutritional value). In some cases with genu varum deformity a radiograph was available and allowed the diagnosis of Blount disease, suggesting to perform an hypercorrection because the risk of recurrence. When the genu valgum is unilateral and severely advanced, it may induce a secondary contralateral varus deformity, producing a typical bayonet 
deviation (figure 7). A valgus deviation is corrected by a femoral supracondylar osteotomy; a varus deviation is corrected by a tibial infratuberosital and fibular osteotomy. The correction is maintained with cast. Twenty cases only were reviewed out of 80 osteotomies, with satisfying results, but not allowing statistical analysis.
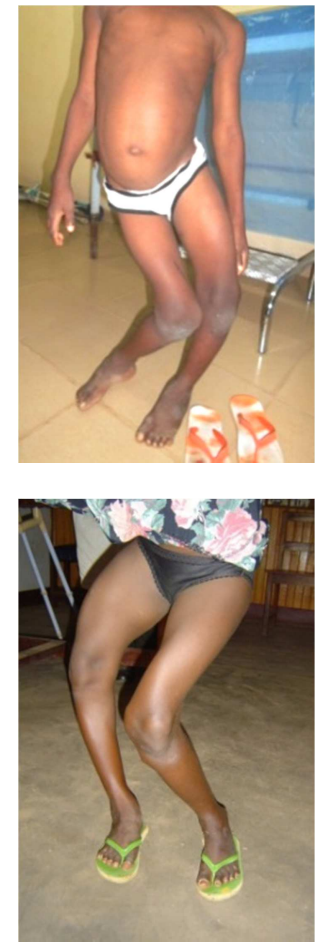

Figure 7. Bilateral deviation of the lower limbs in bayonet. The valgus deformity seems to be the primary one, the contralateral varus being secundary.

\subsection{Sequelae of Cerebral Lesions}

Neurologic sequelae are frequent, including cerebral palsy due to perinatal brain damage, sequelae of neuromalaria frequently associated with mental deficit, and in a few cases sequelae due to a chronic poisoning by poorly processed bitter cassava or Konzo disease [18-20]. Bitter cassava contains a cyanoglucoside linamarin and shortcuts in processing allow large amounts of cyanogens to remain in the cassava; moreover when diets are not supplemented with proteins the children are not able to sufficiently detoxify the cyanide, consequently developing symptoms of upper motoneuron damage. Clinical picture is an abrupt onset of isolated and symmetric spastic paraparesia of the lower limbs, no progressive but permanent. In all these neurologic lesions, walking may be improved by tenotomies, but remains usually dependant on crutches or sticks.

\subsection{Sequelae of Sepsis}

Various sequelae may be observed after sepsis of bones or joints. A chronic osteitis with endless suppuration is easy to diagnose particularly when a radiograph is available, showing a sequestreum, with complete healing after removal of the sequestreum. Correction of limbs inequality due to a diaphyseal gap or due to a sterilisation of the growth plate requires specific facilities and long treatment, and was not attempted. Ankylosis of the knee or ankle joints in incorrect position can be corrected, but correction of a hip ankylosis in an incorrect position is difficult because of the lack of osteosynthesis.

Surgery has infrequently been considered in sequelae of trauma, because the deformities were moderate with a good prognosis for spontaneous correction or sometimes because of the lack of technical means.

\section{Conclusion}

Paediatric orthopaedics is a specialty which is seldom practised in developing countries; this can be explained by several reasons, including the system of recovery cost, which compels the families to finance the entire cost of their children care. Furthermore the families often consider their child's handicap more as a fatality than as a curable disease, and they leave the child to his fate. Nevertheless the needs are significant; the lesions of the locomotor system, either congenital or acquired, are frequent and often quite incapacitating. Once well informed about the procedure and about the expected result, the families get confidence and agree to the treatment and to contribute to the expenses. Last, but not the least, not all, but many of the disabilities observed in paediatric orthopaedic pathology can be treated by relatively simple protocols, attainable in basic environment conditions, and at low cost.

\section{Conflicts of Interest}

No conflicts of interest

\section{Acknowledgements}

Special thanks should be expressed to all our local partners for their close and continuous cooperation, and especially to the missionaries who day after day take care of the children. This work would have been impossible without their invaluable help.

\section{References}

[1] Cross AB (1977) Crawling patterns in neglected poliomyelitis in Solomon Islands. J Bone Joint Surg [Br] 59: 428-32.

[2] Onimus M, Mandaba JL (1992) Les possibilités de verticalisation et de marche chez l'enfant porteur de séquelles de poliomyélite en milieu africain (Walking in Children after Poliomyelitis). Intern Orthop (SICOT) 16: 196-201.

[3] Ponseti IV (1992) Treatment of congenital club foot. J Bone Joint Surg (Am) 74: 448-454.

[4] Dobbs MD, Rudzki JR, Purcell DB, Walton T, Porter KE, Gurnett CA (2004) Factors predictive of outcome after use of the Ponseti method for the treatment of idiopathic club foot. J Bone Joint Surg (Am) 86-A: 22-27. 
[5] Alves K, Penny N, Kobusingye O, Olupot R, Katz JN, Sabatini CS (2018) Paediatric Musculoskeletal Disease in Kumi District, Uganda: A Cross-Sectional Survey. Int Orthop. 42: 1967-1973.

[6] Onimus M, Brunet L, Gaudeuille F, Issa-mapouka A (2007) Le traitement des séquelles d'injections intramusculaires de sels de quinine en milieu africain. Med Trop 67: 267-273.

[7] Judet J, Judet R, Lagrange J (1956) Une technique de libération de l'appareil extenseur dans les raideurs du genou. Mem Acad Chir 82: 944-7.

[8] Gbenou A S, Kpadonou GT, Fiogbe A M, Zoumenou E, Alao MJ (2013) Iatrogenic Retractile Quadriceps Fibrosis Within Children in Benin: Epidemiological, Clinical, Therapeutical Aspects Afr J Paediatr Surg 10: 211-6.

[9] Sengupta S (1985) Pathogenesis of infantile quadriceps fibrosis ans its correction by proximal release. J Pediatr Orthop 5: 187-91.

[10] Soumah MT, Sylla AL, Toure MR et all (2003) Fibrose quadricipitale après injections intramusculaires dans la cuisse: à propos de 92 cas à l'Hôpital central universitaire Ignace Deen à Conakry. Med Trop 63: 49-52.

[11] Fiogbe AM, Gbenou AS, Magnidet ER,. Biaou O (2013). Distal quadricepsplasty in children: 88 cases of retractile fibrosis following intramuscular injections treated in Benin. Orthopaedics \& Traumatology: Surgery \& Research 99: 817822 .

[12] Hung NN (2011) Analysis of Two Different Techniques in the Treatment of Knee Stiffness in Swing Phase Due to Fibrous
Rectus Femoris Muscle in Children. J Pediatr Orthop B 20: 164-72.

[13] Markus AF, Delaire J. (1993) Functional primary closure of the cleft lip. British Journal of oral and maxillofacial surgery 31: 281-291.

[14] Precious DS 2 (009) Primary bilateral cleft lip/nose repair using the "Delaire" technique. Atlas Oral Maxillofac Surg Clin North Am. 17: 137-46.

[15] Kuna SK, Srinath N, Naveen B S, Hasan K. (2016) Comparison of Outcome of Modified Millard's Incision and Delaire's Functional Method in Primary Repair of Unilateral Cleft Lip: A Prospective Study J Maxillofac Oral Surg 15: 221-8.

[16] Fufa DT, Chuang SS, Yang JY J (2014) Postburn Contractures of the Hand. Hand Surg Am 39: 1869-76.

[17] Brown M, Chung KC (2017) Postburn Contractures of the Hand. Hand Clin 33: 317-331.

[18] Tshala-Katumbay D, Eeg-Olofsson KE, Tylleskär T, KazadiKayembe T. (2001) Impairments, disabilities and handicap pattern in konzo--a non-progressive spastic para/tetraparesis of acute onset. Disabil Rehabil. 23: 731-6.

[19] Boivin MJ, Okitundu D, Makila-Mabe Bumoko G, Sombo MT, Mumba D et all (2013) Neuropsychological effects of konzo: a neuromotor disease associated with poorly processed cassava. Pediatrics 131: 1231-9.

[20] Kashala-Abotnes E, Okitundu D, Mumba D, Boivin MJ, Tylleskär T, Tshala-Katumbay D (2019) Konzo: A Distinct Neurological Disease Associated With Food (Cassava) Cyanogenic Poisoning. Brain Res Bull 145: 87-91. 